\title{
Establishment of a Malignant Glioma Model in Rats
}

\author{
Tae-Wook Song ${ }^{1}$, Jung-Kil Lee ${ }^{1,3}$, Sun-Young Lee', Sen Lian", \\ Sung-Pil Joo', Hyung-Seok Kim ${ }^{2,3}$ \\ Departments of ${ }^{l}$ Neurosurgery, ${ }^{2}$ Forensic Medicine, Chonnam National University Medical School \& \\ Research Institute of Medical Sciences, Gwangju, \\ ${ }^{3}$ The Brain Korea 21Project, Center for Biomedical Human Resources, Chonnam National University, Gwangju, Korea
}

\begin{abstract}
Objective: Matrix metalloproteinases (MMPs), especially MMP-2 and MMP-9 have been known to play an important role in glioma cell invasion. The aim of this study was to establish an experimental primary malignant brain tumor model using C6 glioma cells that can be successfully adapted to rats, and to investigate the expression of MMP-2 and MMP-9.

Methods: The author implanted C6 glioma cells into the brain of male Sprague-Dawley rats, weighing between 250 and $300 \mathrm{~g}$ (aged 7 weeks). Animals in the control group model received $5 \mu$ of phosphate-buffered saline (PBS) instead of C6 glioma cell. Development of tumor, tumor volumes and the expression of MMP-2 and MMP-9 was investigated. Behavioral changes were assessed by the rotarod test after $\mathrm{C} 6$ glioma cell implantation. The lesion was observed grossly in the cerebral cortex at 1 week after surgery.

Results: On hematoxylin-eosin (H \& E) staining, increased cellularity and cellular atypia were observed in the C6 glioma cell implanted brain. Immunohistochemistry $(\mathrm{IHC})$ study revealed high expression of MMP-2 and MMP-9, and estimated tumor volumes were increased time-dependently. The rotarod test showed that motor ability of rats implanted with $\mathrm{C} 6$ glioma cells reduced over time.

Conclusion: This study demonstrated successful development of an experimental primary glioma model in rats through C6 glioma cell implantation. In IHC study, an increased expression of MMP-2 and MMP-9 was observed in tumor lesion. To identify the relationship between tumor growth and expression of MMPs, further quantitative and biochemical studies are needed.
\end{abstract}

Key Words: Glioma $\cdot$ Rats $\cdot$ Matrix metalloproteinases $\cdot$ Immunohistochemistry

\section{INTRODUCTION}

Cerebral gliomas represent about $40 \%$ to $60 \%$ of primary brain tumors. Current therapeutic strategies comprise mass-reductive surgery followed by radiation and chemotherapy. In spite of these multiple treatment regimens, the prognosis of patients with glioma is bad due to aggressive invasion by glioma cells ${ }^{12}$. Chemotherapy provides some benefits to patients with malignant glioma as it increases the life span moderately compared to surgery and radiation ${ }^{10,11)}$. However, chemotherapy for glioma has certain limitations such as frequent therapeutic failure due to improper delivery and limited/low bioavailability of the drug at the site of brain tumor. For providing adequate treatment, detailed knowledge of the biological features of

Corresponding author: Jung-Kil Lee

Department of Neurosurgery, Chonnam National University Medical School

\& Research Institute of Medical Sciences, 42 Jebong-ro, Dong-gu, Gwangju 61469, Korea

Tel: +82-62-220-6602, Fax: +82-62-224-9865

E-mail: jkl@chonnam.ac.kr glioma should be obtained. For understanding the biological features of glioma, it is necessary to establish an experimental animal model. However, there are some reports of an experimental glioma model ${ }^{3)}$.

Matrix metalloproteinases (MMPs), a family of zinc-dependent proteinases with extracellular matrix (ECM) remodeling and degrading properties, have been shown to be key mediators of cancer invasion ${ }^{1,5)}$. MMPs, which are released by tumor cells or surrounding stroma, facilitate cancer cell invasion, intravasation and extravasation through the degradation of the ECM and basement membranes, ${ }^{3,6}$. MMP-2 and MMP-9 have been widely studied in this regard, as they cleave type IV collagen, which is the predominant component of the basement membrane $^{4)}$. Both have been found to be overexpressed in malignant glioma cells and may facilitate the invasion of tumor cells ${ }^{7,8)}$.

In this study, the author established a comprehensive, reliable, and reproducible animal model of glioma in rats by a single injection of C6 glioma cells through a simplified stereotaxic cannula system and investigated the expression of MMP-2 and MMP-9 associated with glioma cell growth by an immunohistochemistry (IHC) study. 


\section{MATERIALS AND METHODS}

\section{Cell Culture}

Rat C6 glioma cell line was purchased from the American Type Culture Collection (ATCC; Manassas, VA, USA). Cells were grown in $75 \mathrm{~cm}^{2}$ flasks containing $10 \mathrm{~mL}$ of $1 \times \mathrm{RPMI}$ 1640 (Mediatech Inc., Manassas, VA, USA) supplemented with $10 \%$ fetal bovine serum (FBS) (Mediatech Inc.) and 1\% penicillin and streptomycin (Mediatech Inc.) in a fully-humidified incubator containing $5 \% \mathrm{CO}_{2}$ at $37^{\circ} \mathrm{C}$. Cells were serially passaged following trypsinization using a trypsin/ethylenediaminetetraacetic acid (EDTA) solution (Mediatech Inc.).

\section{1) Animals}

Seven-week-old male Sprague-Dawley rats weighing between 250 and $300 \mathrm{~g}$ were used in the present study. All animal care, experiments, and euthanasia were performed in accordance with protocols approved by the Chonnam National University Animal Care and Committee. The rats were kept in $12 \mathrm{hr}$ light/ dark cycle, and allowed free access to food and water.

\section{2) Surgery for Intracerebral Tumor Cell Implantation}

Each rat was anesthetized with 5\% isoflurane and maintained on 2\% isoflurane in an oxygen/air mixture using gas anesthesia in a stereotaxic frame (Stoelting, Wood Dale, IL, USA). After achieving a deep level of anesthesia, the rat was placed in a prone position with a warmed surgical pad to preserve body temperature throughout the procedure. The rectal temperature was controlled during surgery at $37 \pm 0.5^{\circ} \mathrm{C}$ with a homeothermic blanket (Harvard Apparatus, Holliston, MA, USA). All surgical procedures were carried out using autoclaved or sterile instruments in a clean environment. Prior to surgery, the animal was covered with a sterile drape except for the operative area in the brain, which was decontaminated by applying sufficient betadine solution with a cotton tipped applicator. A midline scalp incision was made to expose the coronal suture. A hole was made in the cranial bone $4 \mathrm{~mm}$ to the right of the bregma and $1 \mathrm{~mm}$ anterior to the coronal suture by a mini-drill.

C6 glioma cells were prepared fresh from culture to ensure optimal viability of cells during tumor inoculation. A microinfusion pump (Microdialysis CMA/100, Solna, Sweden) was used for accurate and even delivery of tumor cells. Cells at a concentration of $1.0 \times 10^{6}$ units in $5 \mu \mathrm{L}$ of phosphate-buffered saline (PBS) were injected at an infusion rate of $1 \mu \mathrm{l} / \mathrm{min}$. After injection of the tumor cells, the needle was kept in place for about 3 min for equilibration of pressure within the cranial vault. Similarly, the negative controls received $5 \mu \mathrm{L}$ of PBS instead of $\mathrm{C} 6$ glioma cell implantation. The animals recovered from anesthesia, were returned to the animal care facilities and resumed their normal activity in cages. After 1, 2, and 3 weeks following tumor cell implantation, all of the animals were sacrificed and evaluated for glioma development.

\section{Histopathological Study}

The brains were then removed at 7, 14, and 21 days after surgery under anesthetic state with isoflurane. The brains were fixed in the same solution overnight at room temperature. The brains were cut into cross or longitudinal sections and processed for paraffin embedding. Representative sections were sliced into $4 \mu \mathrm{m}$ thick sections and stained with hematoxylin$\operatorname{eosin}(\mathrm{H} \& \mathrm{E})$.

IHC analysis of the antibodies for MMP-2 and MMP-9 was performed (AbCam, Cambridge, MA, USA; Dilution 1: 1,000). Immunostaining was performed by the routine avidin-biotin complex $(\mathrm{ABC})$ method. Briefly, representative paraffin blocks were consecutively cut into $4 \mu \mathrm{m}$ thick samples, and IHC staining was carried out using the Microprobe Immuno/DNA stainer (Fisher Scientific Co., Pittsburgh, PA, USA). The sections were deparaffinized in xylene, and treated with $0.3 \%$ hydrogen peroxide in methanol for $20 \mathrm{~min}$ to block endogenous peroxidase activity. The sections were subjected to heat in a pressure cooker in $10 \mathrm{mM}$ citrate-phosphate buffer ( $\mathrm{pH}$ 6.0), then incubated with the antibodies overnight at $4{ }^{\circ} \mathrm{C}$. Anti-mouse immunoglobulin G(Sigma-Aldrich, St. Louis, MO, USA) labeled with biotin was used as a secondary antibody for the detection of primary antibodies, and it was incubated for $7 \mathrm{~min}$ at $45^{\circ} \mathrm{C}$. The streptavidin-horseradish peroxidase (Research Genetics, Huntsville, AL, USA) detection system was applied to capillary channels, followed by $10 \mathrm{~min}$ of incubation at $45^{\circ} \mathrm{C}$. After drainage, the tissue sections were ready for chromogen reaction with $0.02 \%$ diaminobenzidine. The sections were counterstained with hematoxylin and mounted with Universal Mount (Research Genetics). Negative controls were treated similarly except that the primary antibodies were omitted. Each image was captured by a JVC Digital Camera system (JVC Digital Camera KY-F70B, JVC Corporation, Tokyo, Japan) mounted on a Nikon microscope (Nikon E600; Nikon Company, Tokyo, Japan) at $\times 200$ magnification.

\section{Estimation of Tumor Volume}

Tumor volumes $\left(\mathrm{mm}^{3}\right)$ were estimated using the formula of $\mathrm{L} \times \mathrm{H} \times \mathrm{W} \times \pi / 6$, where $\mathrm{L}$ is the length, $\mathrm{W}$ is the width, and $\mathrm{H}$ is the height of the tumor in millimeters. Tumor volume estimation was performed at 7, 14, and 21 days after implantation. 


\section{Behavior Test}

Motor impairment was assessed by the accelerating rotarod test. A 5 min activity test was performed in each rat. Prior to the induction of tumor, the rats were trained on the rotarod for 3 consecutive days, for a total 9 sessions. The rotating drum was accelerated from 4 to $40 \mathrm{rpm}$ over $5 \mathrm{~min}$, and the latency, in sec, for the animal to fall off the drum was recorded. Each session included three consecutive trials, with a maximum time of 300 sec; the mean fall latency was calculated from the three trials. Behavioral testing was conducted at 3, 7, 14, and 21 days after surgery.

\section{RESULTS}

\section{Histopathological Features of Intracerebral C6 Glioma}

Under light microscopy (×200 magnification), H \& E staining of brain sections implanted with C6 glioma cells showed distinct morphological features that were different from those in the control group. Inside the tumor area, a greater accumulation of cell mass was found with fibrillary arrangements, numerous mitoses, and cellular atypia. These characteristics are consistent with a high-grade astrocytic tumor; however, these features were not found in the brain sections of the negative control group (Fig. 1).

\section{Gross Findings and Estimation of Tumor Volume}

On gross examination, the brains at one week after tumor cell implantation showed a minimal lesion, but the brains at two week after implantation showed a definite tumor lesion. In the brains at three week after tumor cell implantation, the tumor lesion had invaded $1 / 3$ of the cerebral hemisphere (Fig. 2). As expected, the tumor volume increased over time (Fig. 3).

\section{Behavior Test}

The rotarod test showed that the motor ability of the rats implanted with C6 glioma cells reduced over time. In future studies, this data can be used for comparison with the treatment group to detect the effect on tumor control (Fig. 4).

\section{IHC}

The study showed high expression of MMP-2 by IHC in

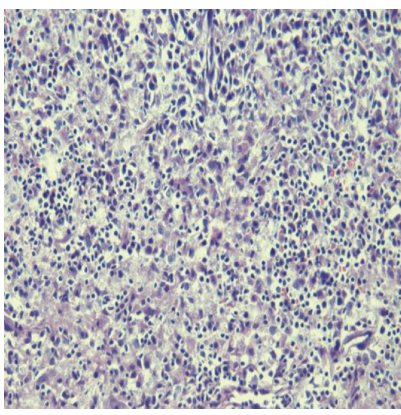

Fig. 1. The results of hematoxylineosin ( $\mathrm{H} \& \mathrm{E})$ staining. Brain section of tumor cell implanted group; inside the lesion of cell implanted brain section, greater accumulation of cell mass was found with fibrillary arrangements, numerous mitoses, and cellular atypia.

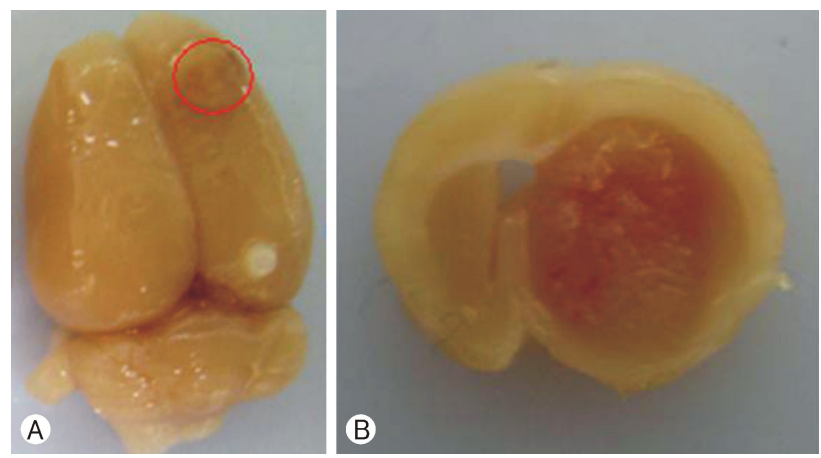

Fig. 2. The gross findings of developed brain tumors. (A, B) At third week after implantation, tumor lesion was invaded up to about $1 / 3$ of brain hemisphere.

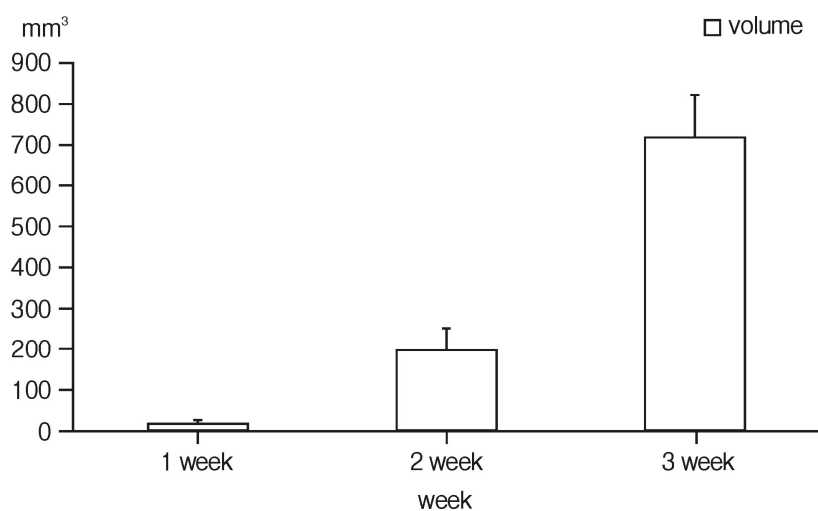

Fig. 3. The tumor volume. Tumor volume was about $12 \mathrm{~mm}^{3}$ at first week after tumor cell implantation. The third week after implantation the tumor volume was increased to about $750 \mathrm{~mm}^{3}$.

brain sections implanted with C6 glioma cells and which showed tumor invasion. Similarly, expression of MMP-9 was also highly detected in brain sections implanted with $\mathrm{C} 6$ glioma cells (Fig. 5).

\section{DISCUSSION}

Glioma is the most common primary tumor of the brain, 


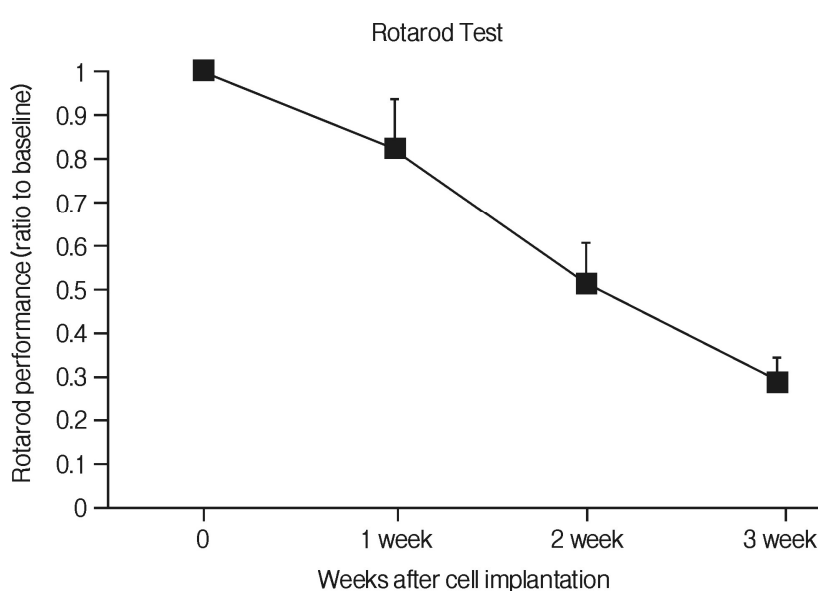

Fig. 4. The result of rotarod test. The results were about 0.82 ratio to baseline at 1 week after surgery. Three weeks after surgery, the values were decreased to about 0.28 ratio to baseline.

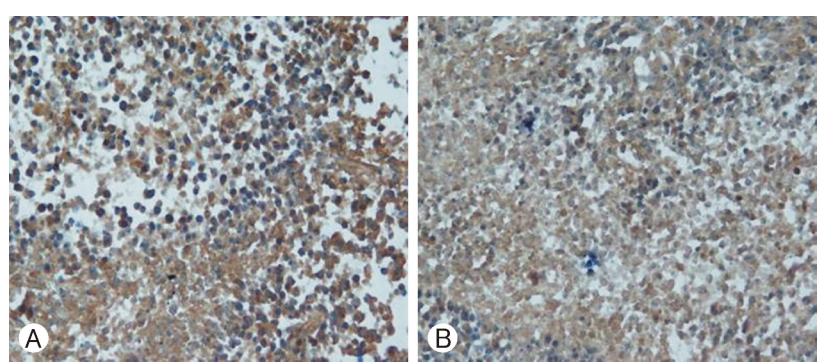

Fig. 5. The results of immunohistochemistry (IHC) study. (A) Tumor cell implanted brain section to matrix metalloproteinase (MMP)-2. (B) Tumor cell implanted brain section to MMP-9. High expression of MMP-2 and MMP-9 by IHC was found in C6 glioma implanted brain sections compared to control group.

and more than one-half of all gliomas exhibit very aggressive and malignant features. In spite of aggressive multimodal therapy, the median survival of patients with malignant glioma is less than 1 year $^{9,10}$. Therefore, there is a continuing requirement of establishing animal models in experimental neurooncology that can be used to assess the effects of innovative approaches for the treatment of brain gliomas.

For establishing an adequate animal model of glioma, the author implanted C6 glioma cells into the rat brain. Parsa et al. ${ }^{9)}$ reported that the success rate of intracranial tumor cell implantation was $89 \%$ at a tumor cell concentration of $1.0 \times$ $10^{6}$ units, therefore, the author injected a concentration of $1.0 \times 10^{6}$ units of $\mathrm{C} 6$ glioma cells in $5 \mu \mathrm{L}$ of PBS for tumor development. One week after tumor cell implantation, a small lesion was observed only grossly. However, the lesion timedependently increased in size. Three weeks after surgery, the lesion was easily observed on gross examination. On $\mathrm{H} \&$ E staining in the author's animal model, the lesion showed increased cellularity and marked atypia. These findings were compatible with the microscopic findings of cerebral glioma.

The hallmarks of human malignant gliomas are their marked growth and invasiveness. Tumor growth and invasion have been associated with increased ECM degradation, and the proteases play a critical role in this process ${ }^{5,12)}$. Proteinases, particularly MMPs, have recently been identified as mediators of tumor growth, invasion and metastasis ${ }^{1}$. MMPs are a family of zinc-binding proteolytic enzymes that degrade ECM components of the basement membrane. MMPs have been implicated in numerous pathological conditions including atherosclerosis, inflammation, tumor growth, metastasis, and cerebral ischemia. Excessive proteolytic activity of MMPs after tissue injury or development of tumor can be detrimental, and can lead to disruption of the ECM and excessive tumor growth and invasion. Among the MMPs, MMP-2 and MMP-9, which are critical factors in basement membrane degradation, have been known to be the key enzymes associated with tumor growth and invasion ${ }^{5}$. The author compared the immunoreactivity of MMP-2 and MMP-9 between the sham surgery group and the $\mathrm{C} 6$ glioma cell implantation group. The author found a strong immunoreactivity in tumor tissue slides compared to that in slides from the sham surgery group. This indicates that MMP-2 and MMP-9 might be overexpressed in the tumor tissue from C6 rat glioma model. Forsyth et al. ${ }^{1)}$ reported that MMP-1 and MMP-2 may be involved in both glial cell invasion and angiogenesis. In the future, a quantitative study assessing the expression and activity of MMP-2 and MMP-9 through western blotting and gelatin zymography should be performed. If the relationship between growth of C6 glioma cells and the expression of MMPs is well established, the therapeutic effects of newly innovated agents or methods on tumor growth can be evaluated using the C6 rat glioma model established by the author.

Appropriate behavioral testing is needed for the evaluation of potential therapeutic interventions for glioma in an animal model. The rotarod test for motor performance provides quantitative, objective, and reproducible measures of functional impairment in a rat animal model ${ }^{2}$. This test has the sensitivity to track behavioral recovery over time. Therefore, it is very useful for comparing the neurologic status at different time points after intracerebral tumor cell implantation and for comparing the effects of several innovative therapeutic agents or methods. The rotarod test is a well-established procedure for testing balance and coordination aspects of motor performance. The only drawback of this test is the requirement of extensive training over a number of sessions before surgery in the animal. In this study, the glioma lesion increased time-dependently after tumor cell implantation. On the other hand, the time required for performing the rotarod test was gradually decreased. Firstly, it is necessary to obtain confirmation of neuro- 
logic improvement and that of a decrease in tumor size; however, previously mentioned findings indicate that researchers can investigate the efficacy of new therapeutic agents by using the animal model established by the author and the rotarod test.

\section{CONCLUSION}

The author successfully developed an experimental primary glioma model in rats by performing C6 glioma cell implantation. Tumor size was increased time-dependently, and the time required for performing the rotarod test was also decreased timedependently. A strong expression of MMP-2 and MMP-9 was observed through an IHC study in the experimental group compared to the sham surgery group. To identify the relationship between tumor growth and expression of MMPs, further quantitative and biochemical studies are needed.

\section{ACKNOWLEDGEMENTS}

This work was supported by a research grant from the Research Institute of Medical Sciences, Chonnam National University.

\section{REFERENCES}

1. Forsyth PA, Wong H, Laing TD, Rewcastle NB, Morris DG, Muzik H, et al.: Gelatinase-A (MMP-2), gelatinase-B (MMP-9) and membrane type matrix metalloproteinase-1 (MT1-MMP) are involved in different aspects of the pathophysiology of malignant gliomas. Br J Cancer 79:1828-1835, 1999
2. Hunter AJ, Hatcher J, Virley D, Nelson P, Irving E, Hadingham SJ, et al.: Functional assessments in mice and rats after focal stroke. Neuropharmacology 39:806-816, 2000

3. Jallo GI, Volkov A, Wong C, Carson BS, Sr., Penno MB: A novel brainstem tumor model: functional and histopathological characterization. Childs Nerv Syst 22:1519-1525, 2006

4. Kim SH, Cho NH, Kim K, Lee JS, Koo BS, Kim JH, et al.: Correlations of oral tongue cancer invasion with matrix metalloproteinases (MMPs) and vascular endothelial growth factor (VEGF) expression. J Surg Oncol 93:330-337, 2006

5. Kleiner DE, Stetler-Stevenson WG: Matrix metalloproteinases and metastasis. Cancer Chemother Pharmacol 43 Suppl:S42-S51, 1999

6. MacDougall JR, Matrisian LM: Contributions of tumor and stromal matrix metalloproteinases to tumor progression, invasion and metastasis. Cancer Metastasis Rev 14:351-362, 1995

7. Nakada M, Okada Y, Yamashita J: The role of matrix metalloproteinases in glioma invasion. Front Biosci 8:e261-e269, 2003

8. Nakagawa T, Kubota T, Kabuto M, Sato K, Kawano H, Hayakawa $\mathrm{T}$, et al.: Production of matrix metalloproteinases and tissue inhibitor of metalloproteinases-1 by human brain tumors. J Neurosurg 81:69-77, 1994

9. Parsa AT, Chakrabarti I, Hurley PT, Chi JH, Hall JS, Kaiser MG, et al.: Limitations of the C6/Wistar rat intracerebral glioma model: implications for evaluating immunotherapy. Neurosurgery 47:993-999; discussion 999-1000, 2000

10. Stewart LA: Chemotherapy in adult high-grade glioma: a systematic review and meta-analysis of individual patient data from 12 randomised trials. Lancet 359:1011-1018, 2002

11. Yamini B, Yu X, Gillespie GY, Kufe DW, Weichselbaum RR: Transcriptional targeting of adenovirally delivered tumor necrosis factor alpha by temozolomide in experimental glioblastoma. Cancer Res 64:6381-6384, 2004

12. Zhang X, Li X, Wu JW, Gao DK, Liang JW, Liu XZ: Experiment and observation on invasion of brain glioma in vivo. $\mathrm{J}$ Clin Neurosci 9:668-671, 2002 